\title{
The Pinch Technique Approach to the Physics of Unstable Particles
}

\author{
Joannis Papavassiliou \\ CERN Theory Division, CH-1211 Geneva 23, \\ Switzerland
}

Abstract: The consistent description of unstable particles within the framework of perturbative gauge field theories necessitates the definition and resummation of off-shell Green's functions, which must respect several crucial physical requirements. We present the solution to this problem at one-loop, using the pinch technique.

\section{Resonances and the need for re- summation}

The physics of unstable particles in general [1]. and the computation of resonant transition amplitudes in particular [2] has attracted significant attention in recent years, because it is both phenomenologically relevant and theoretically challenging. In what follows we will formulate the problem in simple terms, before we proceed to its actual solution.

The mathematical expressions for computing transition amplitudes are ill-defined in the vicinity of resonances, because the tree-level propagator of the particle mediating the interaction, i.e. $\Delta=\left(s-M^{2}\right)^{-1}$, becomes singular as the center-of-mass energy $\sqrt{s} \sim M$. The standard way for regulating this physical kinematic singularity is to use a Breit-Wigner type of propagator, which essentially amounts to the replacement $\left(s-M^{2}\right)^{-1} \rightarrow\left(s-M^{2}+i M \Gamma\right)^{-1}$, where $\Gamma$ is the width of the unstable (resonating) particle. The field-theoretic mechanism which enables this replacement is the Dyson resummation of the (one-loop) self-energy $\Pi(s)$ of the unstable particle, which leads to the substitution $\left(s-M^{2}\right)^{-1} \rightarrow$ $\left[s-M^{2}+\Pi(s)\right]^{-1}$; the running width of the particle is then defined as $M \Gamma(s)=\Im m \Pi(s)$, whereas the usual (on-shell) width is simply its value at $s=M^{2}$.
It is well-known that, to any finite order, the conventional perturbative expansion gives rise to expressions for physical amplitudes which are endowed with crucial properties. For example, the amplitudes are independent of the gauge-fixing parameter (GFP) chosen to quantize the theory, they are gauge-invariant (in the sense of current conservation), they are unitary (in the sense of probability conservation), and well behaved at high energies. The above properties are however not always encoded into the individual Green's functions which are the building blocks of the aforementioned perturbative expansion; indeed, the simple fact that Green's functions depend in general explicitly on the GFP, indicates that they are void of any physical meaning. Evidently, when going from unphysical Green's functions to physical amplitudes subtle field-theoretical mechanisms are at work, which implement highly nontrivial cancellations among the various Green's functions appearing at a given order.

The happy state of affairs described above is guaranteed within the framework of the conventional perturbative expansion, provided that one works at a given fixed order. It is relatively easy to realize however that the Breit-Wigner procedure is in fact equivalent to a reorganization of the perturbative series; indeed, resumming the self-energy $\Pi$ amounts to removing a particular piece from each order of the perturbative expansion, since from all the Feynman 
graphs contributing to a given order $n$ we only pick the part that contains $n$ self-energy bubbles $\Pi$, and then take $n \rightarrow \infty$. However, given that a non-trivial cancellation involving the unphysical Green's function is generally taking place at any given order of the conventional perturbative expansion, the removal of one of them from each order may or may not distort those cancellations. To put it differently, if $\Pi$ contains unphysical contributions (which would eventually cancel against other pieces within a given order) resumming it naively would mean that these unphysical contributions have also undergone infinite summation (they now appear in the denominator of the propagator $\Delta$ ). In order to remove them one has to add the remaining perturbative pieces to an infinite order, clearly an impossible task. Thus, if the resummed $\Pi$ happened to contain such unphysical terms, one would finally arrive at predictions for the amplitude close to the resonance which would be plagued with unphysical artifacts. It turns out that, while in scalar field theories and Abelian gauge theories $\Pi$ does not contain such unphysical contributions, this seizes to be true in the case of non-Abelian gauge theories.

The most obvious signal revealing that the conventionally defined non-Abelian self-energies are not good candidates for resummation comes from the simple calculational fact that the bosonic radiative corrections to the self-energies of vector $(\gamma, W, Z)$ or scalar (Higgs) bosons induce a nontrivial dependence on the GFP used to define the tree-level bosonic propagators appearing in the quantum loops. This is to be contrasted to the fermionic radiative corrections, which, even in the context of non-Abelian gauge theories behave as in quantum electrodynamics (QED), i.e., they are GFP independent. In addition, formal field-theoretic considerations as well as direct calculations show that, contrary to the QED case, the non-Abelian Green's functions do not satisfy their naive, tree-level Ward identities (WI's), after bosonic one-loop corrections are included. A careful analysis shows that this fundamental difference between Abelian and non-Abelian theories has far-reaching consequences; the naive generalization of the Breit-Wigner method to the latter case gives rise to Born-improved amplitudes, which do not faithfully capture the underlying dynamics. Most notably, due to violation of the optical theorem, unphysical thresholds and artificial resonances appear, which distort the line-shapes of the resonating particles. In addition, the high energy properties of such amplitudes are altered, and are in direct contradiction to the equivalence theorem (ET) [i]

In order to address these issues, a new approach to resonant transition amplitudes has been developed over the past few years [4ivis

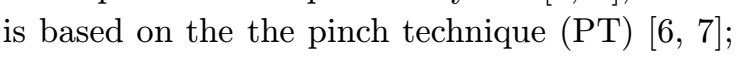
the latter is a diagrammatic method whose main thrust is to exploit the symmetries built into physical amplitudes in order to construct off-shell subamplitudes which are kinematically akin to conventional Green's functions, but, unlike the latter, are also endowed with several crucial properties: (i) they are independent of the GFP; (ii) they satisfy naive (ghost-free) tree-level Ward identities (WI's) instead of the usual Slavnov-Taylor identities; (iii) they display physical thresholds only [4] ; (iv) they satisfy individually the optical and equivalence theorems are analytic functions of the kinematic variables; (vi) the effective two-point functions constructed are universal (process-independent) $\overline{[9} \overline{9}]$, Dyson-

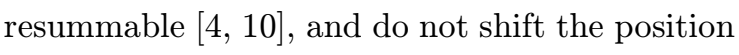
of the gauge-independent complex pole crucial novelty introduced by the PT is that the resummation of graphs must take place only after the amplitude of interest has been cast via the PT algorithm into manifestly physical subamplitudes, with distinct kinematic properties, order by order in perturbation theory. Put in the language employed earlier, the PT ensures that all unphysical contributions contained inside $\Pi$ have been identified and properly discarder, before $\Pi$ undergoes resummation. It is important to emphasize that the only ingredient needed for constructing the PT effective Green's functions is the full exploitation of elementary Ward-identities (EWI), (which are a direct consequence of the BRS [1] in symmetry of the theory) and the proper use of the unitarity and analyticity of the $S$ matrix. In what follows we will describe the method in detail. 


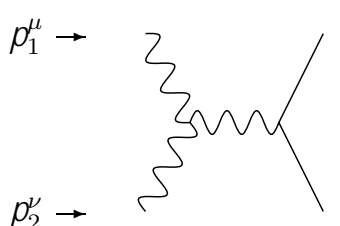

(a)

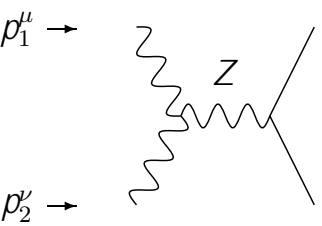

(b)

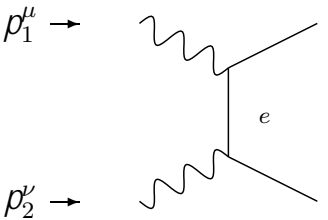

(c)

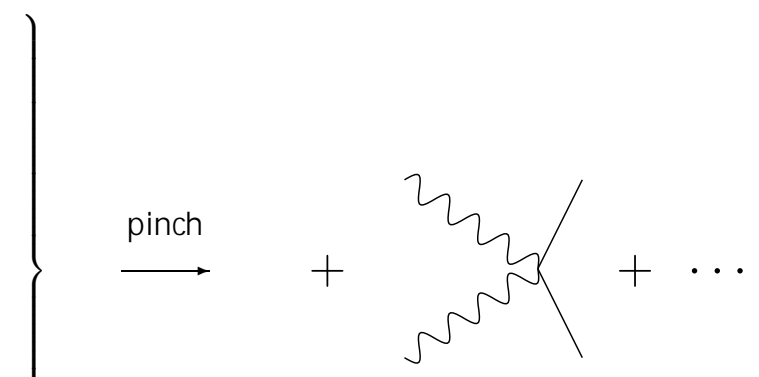

(d)

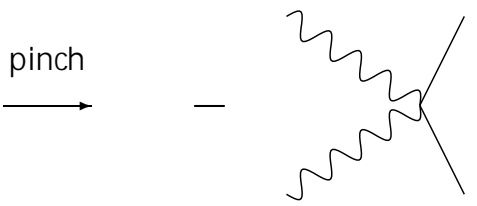

(e)

Figure 1: The fundamental PT cancellation for the process $e^{+} e^{-} \rightarrow W^{+} W^{-}$

\section{The Pinch Technique rearrange- ment of the amplitude.}

Within the PT framework, the transition amplitude $T\left(s, t, m_{i}\right)$ of a $2 \rightarrow 2$ process, can be decomposed as

$T\left(s, t, m_{i}\right)=\widehat{T}_{1}(s)+\widehat{T}_{2}\left(s, m_{i}\right)+\widehat{T}_{3}\left(s, t, m_{i}\right)$,

in terms of three individually g.i. quantities: a propagator-like part $\left(\widehat{T}_{1}\right)$, a vertex-like piece $\left(\widehat{T}_{2}\right)$, and a part containing box graphs $\left(\widehat{T}_{3}\right)$. The important observation is that vertex and box graphs contain in general pieces, which are kinematically akin to self-energy graphs of the transition amplitude. The PT is a systematic way of extracting such pieces and appending them to the conventional self-energy graphs. In the same way, effective gauge invariant vertices may be constructed, if after subtracting from the conventional vertices the propagator-like pinch parts we add the vertex-like pieces coming from boxes. The remaining purely box-like contributions are then also gauge invariant. The way to identify the pieces which are to be reassigned, all one has to do is to resort to the fundamental PT cancella- tion, which is in turn a direct consequence of the elementary Ward identities of the theory. This cancellation is depicted in Fig. for the process $e^{+} e^{-} \rightarrow W^{+} W^{-}$, and will be studied in detail in the next sections.

The PT rearrangement of the amplitude has far-reaching consequences. Perhaps the best way to appreciate them is to study the close connection which exists between gauge invariance and unitarity; the latter is best established by looking at the two sides of the equation for the optical theorem. The optical theorem for a given process $\langle a|T| a\rangle$ is

$$
\Im m\langle a|T| a\rangle=\frac{1}{2} \sum_{f} \int\langle f|T| a\rangle\langle f|T| a\rangle^{*},
$$

where the sum $\sum_{f}$ should be understood to be over the entire phase space and spins of all possible on-shell intermediate particles $m$. The RHS of Eq. (2.2) consists of the product of GFP-independent on shell amplitudes, thus enforcing the gauge-invariance of the imaginary part of the amplitude on the LHS. In particular, even though the LHS contains unphysical particles, such as ghosts and would-be Goldstone bosons, which could give rise to unphysical thresholds, Eq.(12.2) 


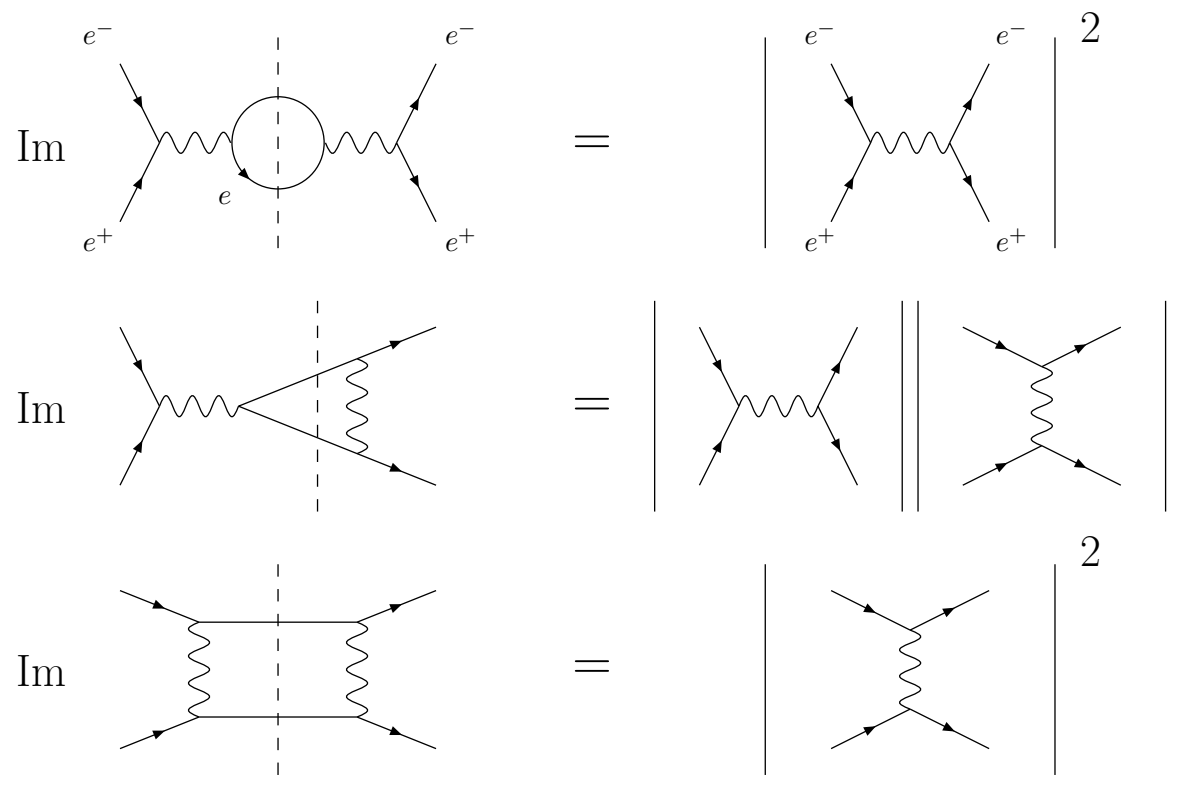

Figure 2: The optical theorem for an Abelian theory

guarantees that all such contributions will vanish. In general, the aforementioned cancellation takes place after contributions from the propagator, vertex-, and box-diagrams have been combined. There are field theories however, such as scalar theories, or QED, which allow for a stronger version of the equality given in Eq. (2.2.): The optical relationship holds individually for the propagator, vertex-, and box-diagrams. In non-Abelian gauge theories however, the afore-mentioned stronger version of the optical theorem does not hold in general. The reason is that unlike their scalar or Abelian counterparts, the conventional selfenergies, vertex and boxes are gauge dependent.

As has been demonstrated in a series of papers [1] however, a strong version of the optical theorem very analogous to that depicted in Fig. 2 can be realized in the context of nonAbelian gauge theories at one loop, if the amplitudes are rearranged according to the PT algorithm. Specifically, let us apply the PT on both sides of Eq. (2.2): The PT rearrangement of the tree-level cross sections appearing in the RHS gives rise to new process-independent (selfenergy-like) parts, which are equal to the imaginary part of the effective self-energies obtained by the application of the PT on the one-loop expression for the amplitude $\langle a|T| b\rangle$ on the LHS .
The same result is true for the vertex- and boxlike parts, defined by the PT on either side of Eq.(2.2). In other words, effective sub-amplitudes obtained after the application of the PT satisfy the optical theorem individually, e.g.,

$\Im m\left(\langle a|T| a\rangle_{\mathrm{PT}}^{j}\right)=\frac{1}{2} \sum_{f} \int\left(\langle f|T| a\rangle\langle f|T| a\rangle^{*}\right)_{\mathrm{PT}}^{j}$,

where the subscript "PT" indicates that the PT rearrangement has been carried out, and the index $j=S, V, B$, distinguishes between effective self-energy, vertex, and boxes, respectively.

Turning to the question of how a resonant amplitude should be regulated, the strategy is now clear: We begin from the RHS of the optical relation given in Eq. (2.2i). The RHS involves onshell physical processes, which satisfy the EWIs. The full exploitation of those EWIs leads unambiguously to a decomposition of the tree-level amplitude into propagator-, vertex- and box-like structures. The propagator-like structure corresponds to the imaginary part of the effective propagator under construction. By imposing the additional requirement that the effective propagator be an analytic function of $q^{2}$ one arrives at a dispersion relation, which, up to renormalizationscheme choices, leads to a unique result for the 


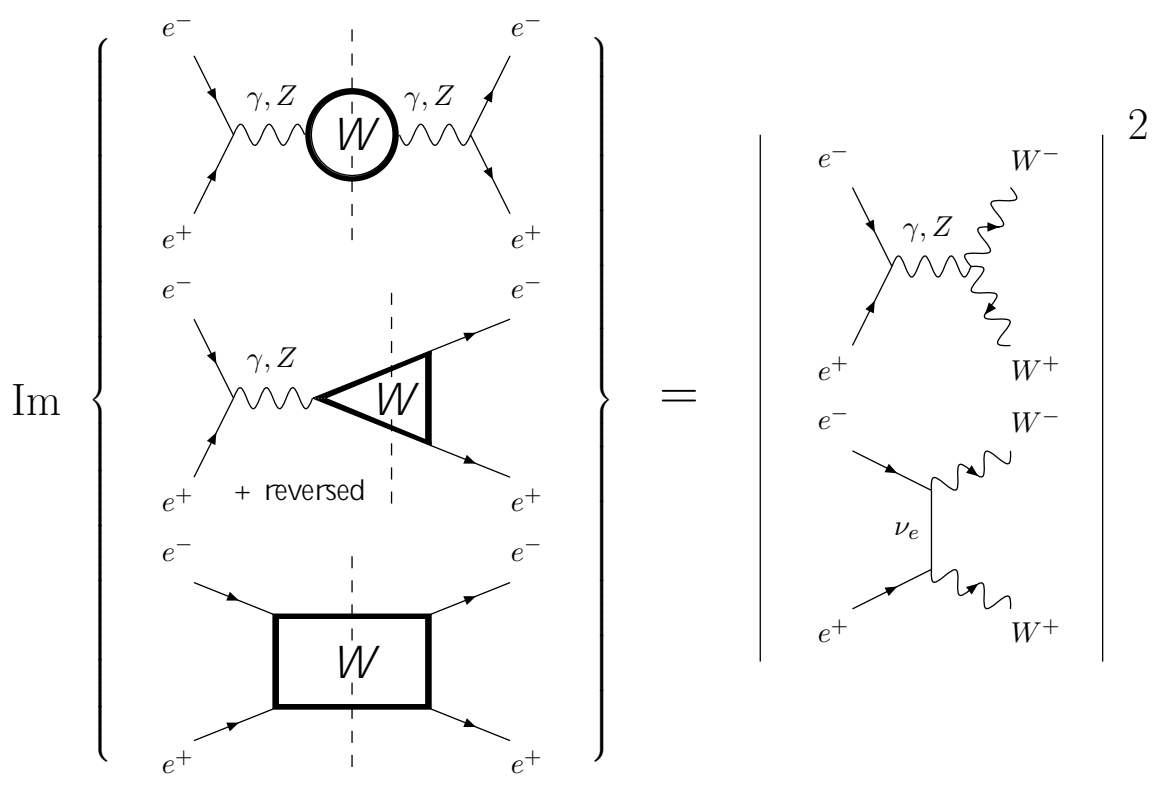

Figure 3: The optical theorem for a non-Abelian theory before the implementation of the PT rearrangement

real part. In the next three section we will study exactly how this strategy is implemented. First we will study the relevant field-theoretical aspects in the case of QCD, which, even though does not allow for (fundamental) resonances, captures most of the issues one needs to understand. Then we will address the electroweak case, and finally we will turn to the particularities of the Higgs-boson resonance.

\section{The case of QCD}

Consider the forward scattering process $q \bar{q} \rightarrow q \bar{q}$, shown in Figure 4. From the optical theorem, we then have

$\Im m\langle q \bar{q}|T| q \bar{q}\rangle=\frac{1}{2}\left(\frac{1}{2}\right) \int d X_{L I P S}|\langle q \bar{q}|T| g g\rangle|^{2}$.

In Eq. (3.1), the statistical factor $1 / 2$ in parentheses arises from the fact that the final on-shell gluons should be considered as identical particles in the total rate. The integration measure $d X_{L I P S}$ denotes the two-body Lorentz invariant phase-space. We now set $\mathcal{M}=\langle q \bar{q}|T| q \bar{q}\rangle$ and $\mathcal{T}=\langle q \bar{q}|T| g g\rangle$, and focus on the RHS of Eq. $(3.1)$

Diagrammatically, the amplitude $\mathcal{T}$ consists of two distinct parts: $t$ and $u$-channel graphs that contain an internal quark propagator, $\mathcal{T}_{t \mu \nu}^{a b}$, as shown in Figs. 3(a) and 3(b), and an s-channel amplitude, $\mathcal{T}_{s \mu \nu}^{a b}$, which is given in Fig. $3(\mathrm{c})$. The subscript " $s$ " and " $t$ " refers to the corresponding Mandelstam variables, i.e. $s=q^{2}=\left(p_{1}+p_{2}\right)^{2}=$ $\left(k_{1}+k_{2}\right)^{2}$, and $t=\left(p_{1}-k_{1}\right)^{2}=\left(p_{2}-k_{2}\right)^{2}$. Defining

$$
V_{\rho}^{c}=g \bar{v}\left(p_{2}\right) \frac{\lambda^{c}}{2} \gamma_{\rho} u\left(p_{1}\right),
$$

we have that

$$
\mathcal{T}_{\mu \nu}^{a b}=\mathcal{T}_{s \mu \nu}^{a b}(\xi)+\mathcal{T}_{t \mu \nu}^{a b},
$$

with

$$
\begin{aligned}
\mathcal{T}_{s \mu \nu}^{a b}(\xi)= & -g f^{a b c} \Delta_{0}^{(\xi), \rho \lambda}(q) \Gamma_{\lambda \mu \nu}\left(q,-k_{1},-k_{2}\right) V_{\rho}^{c}, \\
\mathcal{T}_{t \mu \nu}^{a b}= & -i g^{2} \bar{v}\left(p_{2}\right)\left(\frac{\lambda^{b}}{2} \gamma^{\nu} \frac{1}{\not p_{1}-\not k_{1}-m} \frac{\lambda^{a}}{2} \gamma^{\mu}+\right. \\
& \left.\frac{\lambda^{a}}{2} \gamma^{\mu} \frac{1}{\not p_{1}-\not k_{2}-m} \gamma^{\nu} \frac{\lambda^{b}}{2}\right) u\left(p_{1}\right),
\end{aligned}
$$

where

$$
\begin{aligned}
\Gamma_{\lambda \mu \nu}\left(q,-k_{1},-k_{2}\right)= & \left(k_{1}-k_{2}\right)_{\lambda} g_{\mu \nu}+\left(q+k_{2}\right)_{\mu} g_{\lambda \nu} \\
& -\left(q+k_{1}\right)_{\nu} g_{\lambda \nu}
\end{aligned}
$$

Notice that $\mathcal{T}_{s}$ depends explicitly on the GFP $\xi$, through the tree-level gluon propagator $\Delta_{0 \mu \nu}^{(\xi)}(q)$, 


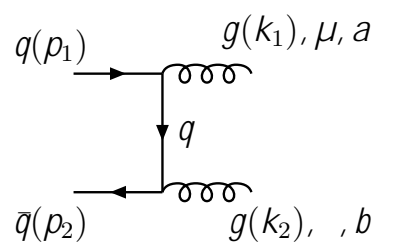

(a)

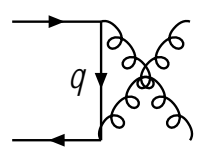

(b)

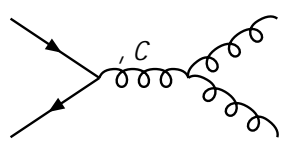

(c)

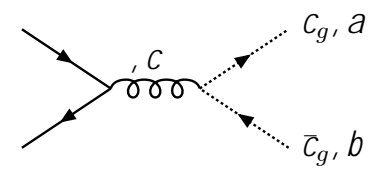

(d)

Figure 4: The diagrams contributing to $\mathcal{T}_{\mu \nu}^{a b}$.

whereas $\mathcal{T}_{t}$ does not. The explicit expression of $\Delta_{0 \mu \nu}^{(\xi)}(q)$ depends on the specific gauge fixing procedure chosen. In addition, we define the quantities $\mathcal{S}^{a b}$ and $\mathcal{R}_{\mu}^{a b}$ as follows:

$$
\begin{aligned}
\mathcal{S}^{a b} & =g f^{a b c} \frac{k_{1}^{\sigma}}{q^{2}} V_{\sigma}^{c} \\
& =-g f^{a b c} \frac{k_{2}^{\sigma}}{q^{2}} V_{\sigma}^{c}
\end{aligned}
$$

and and

$$
\mathcal{R}_{\mu}^{a b}=g f^{a b c} V_{\mu}^{c} .
$$

Clearly,

$$
k_{1}^{\sigma} \mathcal{R}_{\sigma}^{a b}=-k_{2}^{\sigma} \mathcal{R}_{\sigma}^{a b}=q^{2} \mathcal{S}^{a b} .
$$

We then have

$$
\begin{aligned}
\Im m \mathcal{M}= & \frac{1}{4} \mathcal{T}_{\mu \nu}^{a b} P^{\mu \sigma}\left(k_{1}, \eta_{1}\right) P^{\nu \lambda}\left(k_{2}, \eta_{2}\right) \mathcal{T}_{\sigma \lambda}^{a b *} \\
= & \frac{1}{4}\left[\mathcal{T}_{s \mu \nu}^{a b}(\xi)+\mathcal{T}_{t_{\mu \nu}}^{a b}\right] P^{\mu \sigma}\left(k_{1}, \eta_{1}\right) \times \\
& P^{\nu \lambda}\left(k_{2}, \eta_{2}\right)\left[\mathcal{T}_{s_{\sigma \lambda}}^{a b *}(\xi)+\mathcal{T}_{\sigma_{\sigma \lambda}}^{a b *}\right],
\end{aligned}
$$

where the polarization tensor $P^{\mu \nu}(k, \eta)$ is given by

$$
P_{\mu \nu}(k, \eta)=-g_{\mu \nu}+\frac{\eta_{\mu} k_{\nu}+\eta_{\nu} k_{\mu}}{\eta k}+\eta^{2} \frac{k_{\mu} k_{\nu}}{(\eta k)^{2}} .
$$

Moreover, we have that on-shell, i.e., for $k^{2}=0$, $k^{\mu} P_{\mu \nu}=0$. By virtue of this last property, we see immediately that if we write the three-gluon vertex of Eq. (3.5i) in the form

$$
\begin{aligned}
\Gamma_{\lambda \mu \nu}\left(q,-k_{1},-k_{2}\right)= & {\left[\left(k_{1}-k_{2}\right)_{\lambda} g_{\mu \nu}+2 q_{\mu} g_{\lambda \nu}-2 q_{\nu} g_{\lambda \mu}\right] } \\
& +\left(-k_{1 \mu} g_{\lambda \nu}+k_{2 \nu} g_{\lambda \mu}\right) \\
= & \Gamma_{\lambda \mu \nu}^{F}\left(q,-k_{1},-k_{2}\right)+ \\
& \Gamma_{\lambda \mu \nu}^{P}\left(q,-k_{1},-k_{2}\right),
\end{aligned}
$$

the term $\Gamma_{\rho \mu \nu}^{P}$ dies after hitting the polarization vectors $P_{\mu \sigma}\left(k_{1}, \eta_{1}\right)$ and $P_{\nu \lambda}\left(k_{2}, \eta_{2}\right)$. Therefore, if we denote by $\mathcal{T}_{s}^{F}(\xi)$ the part of $\mathcal{T}_{s}$ which survives,

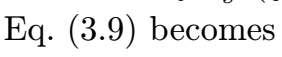

$$
\begin{aligned}
\Im m \mathcal{M}= & \frac{1}{4}\left[\mathcal{T}_{s}^{F}(\xi)+\mathcal{T}_{t}\right]_{\mu \nu}^{a b} P^{\mu \sigma}\left(k_{1}, \eta_{1}\right) \times \\
& P^{\nu \lambda}\left(k_{2}, \eta_{2}\right)\left[\mathcal{T}_{s}^{F}(\xi)+\mathcal{T}_{t}\right]_{\sigma \lambda}^{a b *} \cdot(3.1
\end{aligned}
$$

The next step is to verify that any dependence on the GFP inside the propagator $\Delta_{0 \mu \nu}^{(\xi)}(q)$ of the offshell gluon will disappear. This is indeed so, because the longitudinal parts of $\Delta_{0 \mu \nu}$ either vanish because the external quark current is conserved, or because they trigger the following EWI:

$$
q^{\mu} \Gamma_{\mu \alpha \beta}^{F}\left(q,-k_{1},-k_{2}\right)=\left(k_{1}^{2}-k_{2}^{2}\right) g_{\alpha \beta},
$$


which vanishes on shell. This last EWI is crucial, because in general, current conservation alone is not sufficient to guarantee the GFP independence of the final answer. In the covariant gauges for example, the gauge fixing term is proportional to $q^{\mu} q^{\nu}$; current conservation kills such a term. But if we had chosen an axial gauge instead, i.e.

$$
\Delta_{0 \mu \nu}^{(\tilde{\eta})}(q)=\frac{P_{\mu \nu}(q, \tilde{\eta})}{q^{2}}
$$

where $\tilde{\eta} \neq \eta$ in general, then only the term $\tilde{\eta}_{\nu} q_{\mu}$ vanishes because of current conservation, whereas the term $\tilde{\eta}_{\nu} q_{\mu}$ can only disappear if Eq. ( holds. So, Eq. (3.1.) becomes

$$
\begin{aligned}
\Im m \mathcal{M}= & \frac{1}{4}\left(\mathcal{T}_{s}^{F}+\mathcal{T}_{t}\right)_{\mu \nu}^{a b} P^{\mu \sigma}\left(k_{1}, \eta_{1}\right) \times \\
& P^{\nu \lambda}\left(k_{2}, \eta_{2}\right)\left(\mathcal{T}_{s}^{F}+\mathcal{T}_{t}\right)_{\sigma \lambda}^{a b *}, \quad(3
\end{aligned}
$$

where the GFP-independent quantity $\mathcal{T}_{s}^{F}$ is given by

$$
\mathcal{T}_{s \mu \nu}^{F, a b}=-g f^{a b c} \frac{g^{\rho \lambda}}{q^{2}} \Gamma_{\lambda \mu \nu}^{F}\left(q,-k_{1},-k_{2}\right) V_{\rho}^{c} .
$$

Next, we want to show that the dependence on $\eta_{\mu}$ and $\eta^{2}$ stemming from the polarization vectors disappears. Using the on shell conditions $k_{1}^{2}=$ $k_{2}^{2}=0$, we can easily verify the following EWIs:

$$
\begin{aligned}
k_{1}^{\mu} \mathcal{T}_{s \mu \nu}^{F, a b} & =2 k_{2 \nu} \mathcal{S}^{a b}-\mathcal{R}_{\nu}^{a b} \\
k_{2}^{\nu} \mathcal{T}_{s \mu \nu}^{F, a b} & =2 k_{1 \mu} \mathcal{S}^{a b}+\mathcal{R}_{\mu}^{a b}, \\
k_{1}^{\mu} \mathcal{T}_{t \mu \nu}^{a b} & =\mathcal{R}_{\nu}^{a b} \\
k_{2}^{\nu} \mathcal{T}_{t \mu \nu}^{a b} & =-\mathcal{R}_{\mu}^{a b},
\end{aligned}
$$

from which we have that

$$
\begin{aligned}
k_{1}^{\mu} k_{2}^{\nu} \mathcal{T}_{s \mu \nu}^{F, a b} & =q^{2} \mathcal{S}^{a b}, \\
k_{1}^{\mu} k_{2}^{\nu} \mathcal{T}_{t \mu \nu}^{a b} & =-q^{2} \mathcal{S}^{a b} .
\end{aligned}
$$

Using the above EWIs, it is now easy to check that indeed, all dependence on both $\eta_{\mu}$ and $\eta^{2}$ cancels in Eq. $(\bar{\beta} \cdot \overline{1} \overline{1})$, as it should, and we are finally left with (omitting the fully contracted colour and Lorentz indices):

$$
\begin{aligned}
\Im m \mathcal{M}= & \frac{1}{4}\left[\left(\mathcal{T}_{s}^{F} \mathcal{T}_{s}^{F^{*}}-8 \mathcal{S} \mathcal{S}^{*}\right)\right. \\
& \left.+\left(\mathcal{T}_{s}^{F} \mathcal{T}_{t}^{*}+\mathcal{T}_{s}^{F^{*}} \mathcal{T}_{t}\right)+\mathcal{T}_{t} \mathcal{T}_{t}^{*}\right] \\
= & \Im m \widehat{\mathcal{M}}_{1}+\Im m \widehat{\mathcal{M}}_{2}+\Im m \widehat{\mathcal{M}}_{3} .
\end{aligned}
$$

The first part is the genuine propagator-like piece, the second is the vertex, and the third the box. Employing the fact that

$\Gamma_{\rho \mu \nu}^{F} \Gamma_{\lambda}^{F, \mu \nu}=-8 q^{2} t_{\rho \lambda}(q)+4\left(k_{1}-k_{2}\right)_{\rho}\left(k_{1}-k_{2}\right)_{\lambda}$

and

$$
\begin{aligned}
\mathcal{S S}^{*} & =g^{2} c_{A} V_{\rho}^{c} \frac{k_{1}^{\rho} k_{1}^{\lambda}}{\left(q^{2}\right)^{2}} V_{\lambda}^{c} \\
& =\frac{g^{2}}{4} c_{A} V_{\rho}^{c} \frac{\left(k_{1}-k_{2}\right)^{\rho}\left(k_{1}-k_{2}\right)^{\lambda}}{\left(q^{2}\right)^{2}} V_{\lambda}^{c},
\end{aligned}
$$

where $c_{A}$ is the eigenvalue of the Casimir operator in the adjoint representation $\left(c_{A}=N\right.$ for $\mathrm{SU}(N))$, we obtain for $\Im m \widehat{\mathcal{M}}_{1}$

$$
\begin{aligned}
\Im m \widehat{\mathcal{M}}_{1}= & \frac{g^{2}}{2} c_{A} V_{\mu}^{c} \frac{1}{q^{2}}\left[-4 q^{2} t^{\mu \nu}(q)\right. \\
& \left.+\left(k_{1}-k_{2}\right)^{\mu}\left(k_{1}-k_{2}\right)^{\nu}\right] \frac{1}{q^{2}} V_{\nu}^{c} .
\end{aligned}
$$

This last expression must be integrated over the available two-body phase space; using standard results we arrive at the final expression

$$
\Im m \widehat{\mathcal{M}}_{1}=V_{\mu}^{c} \frac{1}{q^{2}} \Im m \widehat{\Pi}^{\mu \nu}(q) \frac{1}{q^{2}} V_{\nu}^{c},
$$

with

$$
\Im m \widehat{\Pi}_{\mu \nu}(q)=-\frac{\alpha_{s}}{4} \frac{11 c_{A}}{3} q^{2} t_{\mu \nu}(q),
$$

and $\alpha_{s}=g^{2} /(4 \pi)$. The vacuum polarization of the gluon within the PT is given by [6]

$$
\widehat{\Pi}_{\mu \nu}(q)=\frac{\alpha_{s}}{4 \pi} \frac{11 c_{A}}{3} t_{\mu \nu}(q) q^{2}\left[\ln \left(-\frac{q^{2}}{\mu^{2}}\right)+C_{U V}\right] .
$$

Here, $C_{U V}=1 / \epsilon-\gamma_{E}+\ln 4 \pi+C$, with $C$ being some constant and $\mu$ is a subtraction point. In Eq. (B. $\overline{2} \overline{\overline{5}})$, it is interesting to notice that a change of $\mu^{2} \rightarrow \mu^{\prime 2}$ gives rise to a variation of the constant $C$ by an amount $C^{\prime}-C=\ln \mu^{\prime 2} / \mu^{2}$. Thus, a general $\mu$-scheme renormalization yields

$$
\begin{aligned}
\widehat{\Pi}_{T}^{R}(s) & =\widehat{\Pi}_{T}(s)-\left(s-\mu^{2}\right) \Re e \widehat{\Pi}_{T}^{\prime}\left(\mu^{2}\right)-\Re e \widehat{\Pi}_{T}\left(\mu^{2}\right) \\
& =\frac{\alpha_{s}}{4 \pi} \frac{11 c_{A}}{3} s\left[\ln \left(-\frac{s}{\mu^{2}}\right)-1+\frac{\mu^{2}}{s}\right]
\end{aligned}
$$


One can readily see now that $\Re e \widehat{\Pi}_{T}^{R}(s)$ can be calculated by the following double subtracted dispersion relation:

$\Re e \widehat{\Pi}_{T}^{R}(s)=\frac{\left(s-\mu^{2}\right)^{2}}{\pi} \int_{0}^{\infty} d s^{\prime} \frac{\Im m \widehat{\Pi}_{T}\left(s^{\prime}\right)}{\left(s^{\prime}-\mu^{2}\right)^{2}\left(s^{\prime}-s\right)}$.

Inserting Eq. (3. $\overline{2} \overline{4})$ into Eq. ( $3 . \overline{2} \overline{7})$, it is not difficult to show that it leads to the result given in Eq. ( $\left.3 \overline{3} \overline{2} \overline{6_{1}}\right)$, a fact that demonstrates the analytic power of the dispersion relations.

\section{The electroweak case}

In this section, we will show how the same considerations apply directly to the case of the electroweak sector of the SM. We consider the charged current process $e^{-} \nu \rightarrow e^{-} \nu$ and assume that the electron mass $m_{e}$ is non-zero, so that the external current is not conserved. We focus on the part of the amplitude which has a threshold at $q^{2}=M_{W}^{2}$. This corresponds the virtual process $W^{-} \rightarrow W^{-} \gamma$, where $\gamma$ is the photon. From the optical theorem, we have

$\Im m\left\langle e^{-} \nu|T| e^{-} \nu\right\rangle=\frac{1}{2} \int d X_{L I P S}\left|\left\langle e^{-} \nu|T| W^{-} \gamma\right\rangle\right|^{2}$.

We set again $\mathcal{M}=\left\langle e^{-} \nu|T| e^{-} \nu\right\rangle$ and $\mathcal{T}=$ $\left\langle e^{-} \nu|T| W^{-} \gamma\right\rangle$. As in the case of QCD, the amplitude consists of two distinct parts, a part that contains an electron propagator (Fig. 4(a)) and a part that does not, which is shown in Figs. 4(b) and $4(\mathrm{c})$. As before, we denote them by $\mathcal{T}_{t}$ and $\mathcal{T}_{s}\left(\xi_{w}\right)$, respectively. We first define

$$
V_{L}^{\mu}=\frac{g_{w}}{2 \sqrt{2}} \bar{v}\left(p_{2}\right) \gamma^{\mu}\left(1-\gamma_{5}\right) u\left(p_{1}\right)
$$

and

$$
S_{R}=\frac{g_{w}}{2 \sqrt{2}} \frac{m_{e}}{M_{W}} \bar{v}\left(p_{2}\right)\left(1+\gamma_{5}\right) u\left(p_{1}\right) .
$$

Clearly, one has the EWI

$$
q_{\mu} V_{L}^{\mu}=M_{W} S_{R}
$$

The amplitude $\mathcal{T}_{s}$ can the be written down in the closed form

$$
\begin{aligned}
& \mathcal{T}_{s \mu \nu}\left(\xi_{w}\right)=i V_{L}^{\lambda} \Delta_{0 \lambda}^{\left(\xi_{w}\right), \rho}(q) \Gamma_{\nu \rho \mu}^{\gamma W^{-} W^{+}}+ \\
& i S_{R} D_{0}^{\left(\xi_{w}\right)}(q) \Gamma_{\nu \mu}^{\gamma G^{-} W^{+}} \text {, }
\end{aligned}
$$

where $\Gamma_{\nu \rho \mu}^{\gamma W^{-} W^{+}}=e \Gamma_{\nu \rho \mu}\left(-k_{2}, q,-k_{1}\right)$ is the treelevel $\gamma W^{-} W^{+}$vertex and $\Gamma_{\nu \mu}^{\gamma G^{-} W^{+}}=e M_{W} g_{\mu \nu}$ is the tree-level $\gamma G^{-} W^{+}$vertex. In the expression $(4.5)$, we explicitly display the dependence on the GFP $\xi_{w}$. In addition, the amplitude $\mathcal{T}_{t}$ is given by

$$
\mathcal{T}_{t}^{\mu \nu}=\frac{i e g_{w}}{2 \sqrt{2}} \bar{v}\left(p_{2}\right) \gamma^{\mu}\left(1-\gamma_{5}\right) \frac{1}{\not p_{1}-\not k_{2}-m_{e}} \gamma^{\nu} u\left(p_{1}\right) \text {. }
$$

Notice that $\mathcal{T}_{t}^{\mu \nu}$ does not depend on $\xi_{w}$. Denoting by $k_{1}$ the four-momentum of the $W$ and by $k_{2}$ that of the photon, Eq. (4.1) becomes

$$
\Im m \mathcal{M}=\mathcal{T}_{\mu \nu} Q^{\mu \rho}\left(k_{1}\right) P^{\nu \sigma}\left(k_{2}, \eta\right) \mathcal{T}_{\rho \sigma}^{*},
$$

where $P^{\mu \nu}$ is the photon polarization tensor given in Eq. $\left(\bar{s}_{-1} \cdot \overline{1} \overline{1}\right)$, and

$$
Q^{\mu \nu}(k)=-g^{\mu \nu}+\frac{k^{\mu} k^{\nu}}{M_{W}^{2}}
$$

is the $W$ polarization tensor. The polarization tensor $Q^{\mu \nu}(k)$ shares the property that, on shell, i.e., for $k^{2}=M_{W}^{2}, k^{\mu} Q_{\mu \nu}(k)=0$. Furthermore, in Eq. (4.7.), we omit the integration measure $1 / 2 \int d X_{L I P S}$.

$\Delta_{0 \mu \nu}^{\left(\xi_{Q}\right)}(q)=t_{\mu \nu}(q) \frac{1}{q^{2}-M^{2}}-\ell_{\mu \nu}(q) \frac{\xi_{Q}}{q^{2}-\xi_{Q} M^{2}}$

with

$$
t_{\mu \nu}(q)=-g_{\mu \nu}+\frac{q_{\mu} q_{\nu}}{q^{2}}, \quad \ell_{\mu \nu}(q)=\frac{q_{\mu} q_{\nu}}{q^{2}} .
$$

First, we will show how the dependence on the GFP $\xi_{w}$ cancels. To that end, we employ the usual decomposition

$$
\Delta_{0 \mu \nu}^{\left(\xi_{w}\right)}(q)=U_{\mu \nu}(q)-\frac{q_{\mu} q_{\nu}}{M_{W}^{2}} D_{0}^{\left(\xi_{w}\right)}\left(q^{2}\right)
$$

the EWI

$$
\begin{aligned}
& q^{\rho} \Gamma_{\nu \rho \mu}^{\gamma W^{-} W^{+}}\left(-k_{2}, q,-k_{1}\right) Q^{\mu \lambda}\left(k_{1}\right) P^{\nu \sigma}\left(k_{2}, \eta\right)= \\
& M_{W} \Gamma_{\mu \nu}^{\gamma G^{-} W^{+}} Q^{\mu \lambda}\left(k_{1}\right) P^{\nu \sigma}\left(k_{2}, \eta\right)
\end{aligned}
$$

and the EWI of Eq. (4.4. following $\xi_{w}$-independent expression for $\mathcal{T}_{s}^{\mu \nu}$

$$
\begin{aligned}
\mathcal{T}_{s}^{\mu \nu} & =i e V_{L}^{\lambda} U_{\lambda \rho}(q) \Gamma^{\nu \rho \mu}\left(-k_{2}, q,-k_{1}\right) \\
& =i e V_{L}^{\lambda} U_{\lambda \rho}(q) \Gamma^{F, \nu \rho \mu}\left(-k_{2}, q,-k_{1}\right) \\
& =\mathcal{T}_{s}^{F, \mu \nu}
\end{aligned}
$$




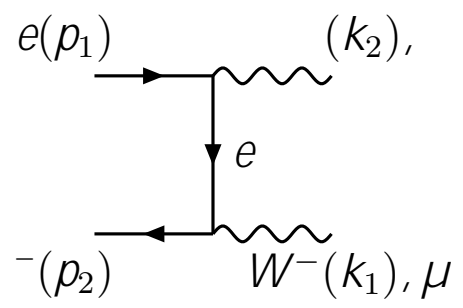

(a)

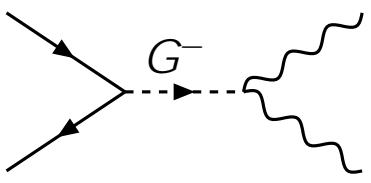

(b)

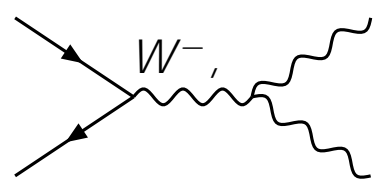

(c)

Figure 5: Amplitudes contributing to the reaction $e^{-} \bar{\nu} \rightarrow W^{-} \gamma$

where contraction over the polarization tensors $Q_{\mu \nu}$ and $P_{\mu \nu}$ is implied. In the last step of Eq. $(\overline{4} . \overline{1} \overline{1})$, we have used the fact that the $\Gamma^{P}$ part of

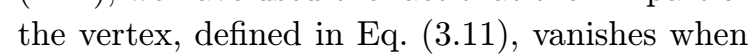
contracted with the polarization tensors.

Next, we show how the dependence on the four-vector $\eta_{\mu}$ and the parameter $\eta^{2}$ vanishes. First, it is straightforward to verify the following EWI:

$$
\begin{aligned}
k_{1}^{\mu} \Gamma_{\nu \rho \mu}^{F}= & {\left[U_{\gamma}^{-1}\left(k_{2}\right)-U^{-1}(q)-U^{-1}\left(k_{1}\right)\right]_{\nu \rho} } \\
& +2 M_{W}^{2} g_{\nu \rho}+\left(k_{1}-k_{2}\right)_{\nu} k_{1 \rho} \\
= & -U_{\nu \rho}^{-1}(q)+2 M_{W}^{2} g_{\nu \rho}- \\
& k_{2 \nu}\left(k_{1}-k_{2}\right)_{\rho}
\end{aligned}
$$

where the on-shell conditions $k_{1}^{2}=M_{W}^{2}$ and $k_{2}^{2}=$ 0 are used in the last equality of Eq. (4.13). Similarly, one has

$$
\begin{aligned}
k_{2}^{\nu} \Gamma_{\nu \rho \mu}^{F}= & {\left[U^{-1}(q)-U^{-1}\left(k_{1}\right)+U_{\gamma}^{-1}\left(k_{2}\right)\right]_{\rho \mu} } \\
& +k_{2 \rho}\left(k_{1}-k_{2}\right)_{\mu} \\
= & U_{\rho \mu}^{-1}(q)-\left(k_{1}-k_{2}\right)_{\rho} k_{1 \mu}
\end{aligned}
$$

with

$$
\begin{aligned}
U_{\alpha \beta}^{-1}(q) & =\left(q^{2}-M_{W}^{2}\right) t_{\alpha \beta}+M_{W}^{2} \ell_{\alpha \beta}, \\
U_{\gamma \quad{ }_{\alpha \beta}}^{-1}(q) & =q^{2} t_{\alpha \beta} .
\end{aligned}
$$

So, when the $\eta^{\sigma} k_{2}^{\nu}$ term from $P_{\nu \sigma}\left(k_{2}, \eta\right)$ gets contracted with $\mathcal{T}_{\mu \nu}$, we have

$$
\begin{aligned}
\eta^{\sigma} k_{2}^{\nu} \mathcal{T}_{s \mu \nu} & =i e \eta^{\sigma} V_{L}^{\lambda}\left[g_{\lambda \mu}-U_{\lambda}^{\alpha}(q) U_{\alpha \mu}^{-1}\left(k_{1}\right)\right] \\
\eta^{\sigma} k_{2}^{\nu} \mathcal{T}_{t \mu \nu} & =-i e \eta^{\sigma} V_{L \mu} .
\end{aligned}
$$

Adding the last two equations by parts, we find

$$
\eta^{\sigma} k_{2}^{\nu} \mathcal{T}_{\mu \nu}=i e \eta^{\sigma} V_{L}^{\lambda} U_{\lambda}^{\alpha}(q) U_{\alpha \mu}^{-1}\left(k_{1}\right)
$$

Since the result is proportional to $k_{1 \mu}$, the fourmomentum of the external $W$ boson, we immediately see that

$$
\eta^{\sigma} k_{2}^{\nu} \mathcal{T}_{\mu \nu} Q^{\mu \rho}\left(k_{1}\right)=0
$$

For the same reasons, the term proportional to $\eta^{2}$ vanishes as well. Consequently, $\Im m \mathcal{M}$ takes on the form

$$
\begin{aligned}
\Im m \mathcal{M}= & -\left(\mathcal{T}_{s}^{F}+\mathcal{T}_{t}\right)_{\mu \nu} Q^{\mu \rho}\left(k_{1}\right)\left(\mathcal{T}_{s}^{F}+\mathcal{T}_{t}\right)_{\rho \nu}^{*} \\
= & \left(\mathcal{T}_{s}^{F}+\mathcal{T}_{t}\right)^{\mu \nu}\left(\mathcal{T}_{s}^{F}+\mathcal{T}_{t}\right)_{\mu \nu}^{*}- \\
& \left(\mathcal{T}_{s}^{F}+\mathcal{T}_{t}\right)^{\mu \nu} \frac{k_{1 \mu} k_{1}^{\rho}}{M_{W}^{2}}\left(\mathcal{T}_{s}^{F}+\mathcal{T}_{t}\right)_{\rho \nu}^{*} \\
= & \Im m \mathcal{M}^{a}+\Im m \mathcal{M}^{b} .
\end{aligned}
$$

The absorptive sub-amplitude, $\Im m \mathcal{M}^{a}$, consists of three terms,

$$
\begin{aligned}
\Im m \mathcal{M}^{a} & =\mathcal{T}_{s}^{F} \mathcal{T}_{s}^{F^{*}}+\left(\mathcal{T}_{s}^{F} \mathcal{T}_{t}^{*}+\mathcal{T}_{t} \mathcal{T}_{s}^{F^{*}}\right)+\mathcal{T}_{t} \mathcal{T}_{t}^{*} \\
& =\Im m \widehat{\mathcal{M}}_{1}^{a}+\Im m \widehat{\mathcal{M}}_{2}^{a}+\Im m \widehat{\mathcal{M}}_{3}^{a}
\end{aligned}
$$




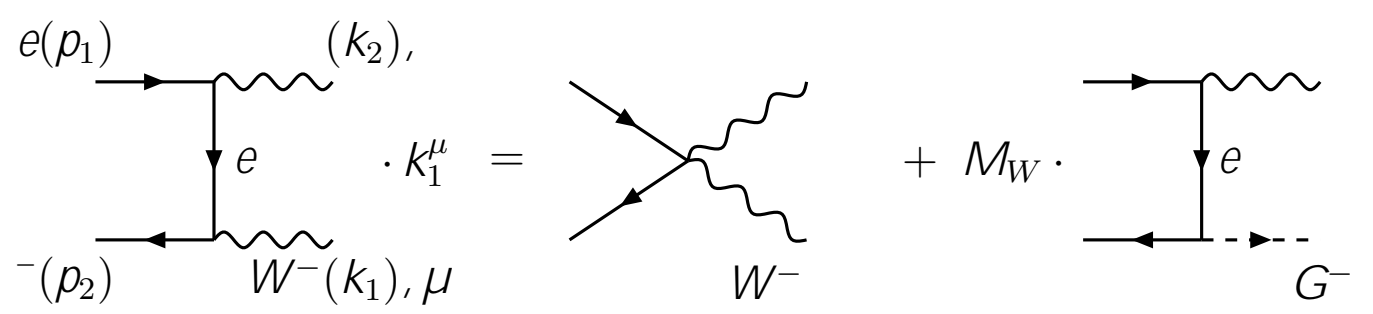

(a)

(b)

(c)

Figure 6: Elementary BRS identity for the $e$-dependent amplitude $\mathcal{T}_{t}^{\mu \nu}$

The first term, $\Im m \widehat{\mathcal{M}}_{1}^{a}$, can easily be identified with a propagator-like contribution. In particu-

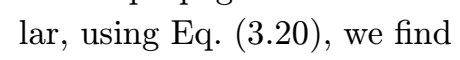

$$
\begin{aligned}
\Im m \widehat{\mathcal{M}}_{1}^{a}= & e^{2} V_{L}^{\rho} U_{\rho \mu}(q)\left[-8 q^{2} t^{\mu \nu}(q)+\times\right. \\
& \left.4\left(k_{1}-k_{2}\right)^{\mu}\left(k_{1}-k_{2}\right)^{\nu}\right] \times \\
& U_{\nu \lambda}(q) V_{L}^{\lambda} .
\end{aligned}
$$

The amplitudes, $\Im m \widehat{\mathcal{M}}_{2}^{a}$ and $\Im m \widehat{\mathcal{M}}_{3}^{a}$, are vertexand box-like contributions, respectively, and they will not be considered any further here.

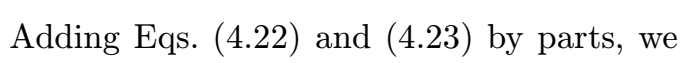
obtain

$$
\begin{aligned}
k_{1}^{\mu}\left(\mathcal{T}_{s}^{F}+\mathcal{T}_{t}\right)_{\mu \nu}= & -i e V_{L \lambda} U^{\lambda \rho}(q) \times \\
& {\left[\left(k_{1}-k_{2}\right)_{\rho} k_{2 \nu}-2 M_{W}^{2} g_{\rho \nu}\right]+M_{W} \mathcal{L}_{\nu} . }
\end{aligned}
$$

Making now use of the EWI of Eq. (44.4) and writing

$$
S_{R}=M_{W} V_{L \mu} U^{\mu \nu}(q) q_{\nu}
$$

We must now isolate the corresponding propagatoxields the following WI for $\mathcal{L}_{\sigma}$ : like piece from $\Im m \mathcal{M}^{b}$. By virtue of the EWI of

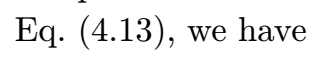

$$
k_{2}^{\nu} \mathcal{L}_{\nu}=-i e S_{R}=-i e M_{W} V_{L \alpha} U^{\alpha \beta}(q) q_{\beta} .
$$

$$
\begin{aligned}
k_{1}^{\mu} \mathcal{T}_{s_{\mu \nu}}^{F}= & -i e V_{L \nu}-i e V_{L \lambda} U^{\lambda \rho}(q) \times \\
& {\left[\left(k_{1}-k_{2}\right)_{\rho} k_{2 \nu}-2 M_{W}^{2} g_{\rho \nu}\right](4}
\end{aligned}
$$

In addition, we evaluate the EWI

$$
\begin{aligned}
k_{1}^{\mu} \mathcal{T}_{t \mu \nu}= & i e V_{L \nu}+M_{W} \frac{i e g_{w} m_{e}}{2 \sqrt{2} M_{W}} \bar{v}\left(p_{2}\right)\left(1+\gamma_{5}\right) \\
& \frac{1}{\not p_{1}-\not k_{2}-m_{e}} \gamma_{\nu} u\left(p_{1}\right) \\
= & i e V_{L \nu}+ \\
& M_{W} \mathcal{L}_{\nu},
\end{aligned}
$$

which is shown diagrammatically in Fig. 5.
Taking the above relations into account, we eventually obtain

$$
\begin{aligned}
\Im m \mathcal{M}^{b}= & -e^{2} V_{L \rho} U^{\rho \mu}(q)\left[4 M_{W}^{2} g_{\mu \nu}+\right. \\
& \left.2\left(k_{1}-k_{2}\right)_{\mu}\left(k_{1}-k_{2}\right)_{\nu}\right] U^{\nu \lambda}(q) V_{L \lambda} \\
& -2 i e M_{W}\left[V_{L \rho} U^{\rho \nu}(q) \mathcal{L}_{\nu}^{*}-\mathcal{L}_{\nu} U^{\nu \lambda}(q) V_{L \lambda}\right] \\
& -\mathcal{L}^{\nu} \mathcal{L}_{\nu}^{*} \\
= & \Im m \widehat{\mathcal{M}}_{1}^{b}+\Im m \widehat{\mathcal{M}}_{2}^{b}+\Im m \widehat{\mathcal{M}}_{3}^{b} .
\end{aligned}
$$

Adding the two propagator-like parts $\Im m \widehat{\mathcal{M}}_{1}^{a}$ and $\Im m \widehat{\mathcal{M}}_{1}^{b}$ from Eqs. $(\underline{4} .2 \overline{1} \bar{\nu})$ and $(\underline{\underline{4}} . \overline{2} \bar{i})$, respectively, 
we find

$$
\begin{aligned}
\Im m \widehat{\mathcal{M}}_{1}= & \Im m \widehat{\mathcal{M}}_{1}^{a}+\Im m \widehat{\mathcal{M}}_{1}^{b} \\
= & e^{2} V_{L}^{\rho} U_{\rho \mu}(q)\left[-8 q^{2} t^{\mu \nu}(q)-4 M_{W}^{2} g^{\mu \nu}\right. \\
& \left.+2\left(k_{1}-k_{2}\right)^{\mu}\left(k_{1}-k_{2}\right)^{\nu}\right] U_{\nu \lambda}(q) V_{L}^{\lambda} .
\end{aligned}
$$

Next, we carry out the phase-space integration over $1 / 2 \int d X_{L I P S}$, using standard integration formulae, we have

$$
\Im m \widehat{\mathcal{M}}_{1}=V_{L \rho} U^{\rho \mu}(q) \Im m \widehat{\Pi}_{\mu \nu}^{W} U^{\nu \lambda}(q) V_{L \lambda}
$$

with

$$
\begin{aligned}
\Im m \widehat{\Pi}_{\mu \nu}^{W}(q)= & \Im m \widehat{\Pi}_{T}^{W}\left(q^{2}\right) t_{\mu \nu}(q)+ \\
& \Im m \widehat{\Pi}_{L}^{W}\left(q^{2}\right) \ell_{\mu \nu}(q) \\
\Im m \widehat{\Pi}_{T}^{W}\left(q^{2}\right)= & \frac{\alpha_{e m}}{2}\left(q^{2}-M_{W}^{2}\right) \times \\
& \left(-\frac{11}{3}+\frac{4 M_{W}^{2}}{3 q^{2}}+\frac{M_{W}^{4}}{3 q^{4}}\right), \\
\Im m \widehat{\Pi}_{L}^{W}\left(q^{2}\right)= & \frac{\alpha_{e m}}{2}\left(q^{2}-M_{W}^{2}\right) \times \\
& \left(-\frac{2 M_{W}^{2}}{q^{2}}+\frac{M_{W}^{4}}{q^{4}}\right) .
\end{aligned}
$$

Here, $\alpha_{e m}=e^{2} /(4 \pi)$ is the electromagnetic fine structure constant. The real part of the transverse, on-shell renormalized, $W$-boson self-energy, $\Re e \widehat{\Pi}_{T}^{W, R}(s)$, can be determined by means of a doubly subtracted dispersion relation. Furthermore, we have to assume a fictitious photon mass, $\mu_{\gamma}$, in order to regulate the infra-red (IR) divergences. More explicitly, the relevant dispersion relation reads

$$
\begin{aligned}
\Re e \widehat{\Pi}_{T}^{W, R}(s)= & \Re e \widehat{\Pi}_{T}^{W}(s)-\left(s-M_{W}^{2}\right) \Re e \widehat{\Pi}_{T}^{W \prime}\left(M_{W}^{2}\right) \\
& -\Re e \widehat{\Pi}_{T}^{W}\left(M_{W}^{2}\right) \\
= & \frac{\left(s-M_{W}^{2}\right)^{2}}{\pi} \\
& \int_{\left(M_{W}+\mu_{\gamma}\right)^{2}}^{\infty} \frac{d s^{\prime} \Im m \widehat{\Pi}_{T}^{W}\left(s^{\prime}\right)}{\left(s^{\prime}-M_{W}^{2}\right)^{2}\left(s^{\prime}-s\right)} .(4.31)
\end{aligned}
$$

To obtain the analytic form of $\Re e \widehat{\Pi}_{T}^{W, R}(s)$, we first evaluate the following integrals:

$$
F_{0}(s)=\left(s-M_{W}^{2}\right) \int_{\left(M_{W}+\mu_{\gamma}\right)^{2}}^{\infty} \frac{d s^{\prime}}{\left(s^{\prime}-M_{W}^{2}\right)\left(s^{\prime}-s\right)}
$$

$$
\begin{aligned}
= & -\ln \left(\frac{\left|s-M_{W}^{2}\right|}{2 M_{W} \mu_{\gamma}}\right), \\
F_{1}(s)= & \left(s-M_{W}^{2}\right) \int_{\left(M_{W}+\mu_{\gamma}\right)^{2}}^{\infty} \frac{d s^{\prime}}{\left(s^{\prime}-M_{W}^{2}\right)\left(s^{\prime}-s\right)} \frac{M_{W}^{2}}{s^{\prime}} \\
= & -\frac{M_{W}^{2}}{s} \ln \left(\frac{\left|s-M_{W}^{2}\right|}{2 M_{W} \mu_{\gamma}}\right)- \\
& \left(1-\frac{M_{W}^{2}}{s}\right) \ln \left(\frac{M_{W}}{2 \mu_{\gamma}}\right), \\
F_{2}(s)= & \left(s-M_{W}^{2}\right) \int_{\left(M_{W}+\mu_{\gamma}\right)^{2}}^{\infty} \frac{d s^{\prime}}{\left(s^{\prime}-M_{W}^{2}\right)\left(s^{\prime}-s\right)} \frac{M_{W}^{4}}{s^{\prime 2}} \\
= & -\frac{M_{W}^{4}}{s^{2}} \ln \left(\frac{\left|s-M_{W}^{2}\right|}{2 M_{W} \mu_{\gamma}}\right)- \\
& \ln \left(\frac{M_{W}}{2 \mu_{\gamma}}\right)+1-\frac{M_{W}^{2}}{s},
\end{aligned}
$$

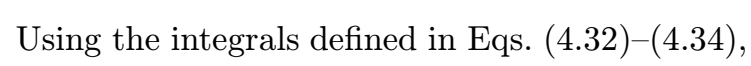
one then obtains

$\Re e \widehat{\Pi}_{T}^{W}(s)=\frac{\alpha_{e m}}{2}\left(s-M_{W}^{2}\right)\left(-\frac{11}{3} F_{0}+\frac{4}{3} F_{1}+\frac{1}{3} F_{2}\right)$.

Eq. ('A. $\left.\bar{A} \overline{3} \overline{y_{1}}\right)$ coincides with the PT $W$-boson selfenergy [12] or equivalently with the $W$-boson selfenergy computed in the background field method [133] for $\xi_{Q}=1$ [1 14 14].

\section{The Higgs boson resonance}

When the center-of-mass (c.m.) energy $\sqrt{s}$ approaches $M_{H}$, amplitudes containing an $s$-channel Higgs boson become singular, and must be regulated. The naive extension of the standard BreitWigner procedure to this case would consist of replacing the free Higgs boson propagator $\Delta_{H}(s)=$ $\left(s-M_{H}^{2}\right)^{-1}$ by a resummed propagator of the form $\left[s-M_{H}^{2}+\Pi^{H H}(s)\right]^{-1}$, where $\Pi^{H H}(s)$ is the one-loop Higgs boson self-energy. However, bosonic radiative corrections induce an additional dependence on the GFP, as one can verify by explicit calculations in a variety of conventional gauges, such as the renormalizable $\left(R_{\xi}\right)$, or axial gauges. Turning to more elaborate gauge fixing schemes does not improve the situation. For example, within the Background Field Method the 


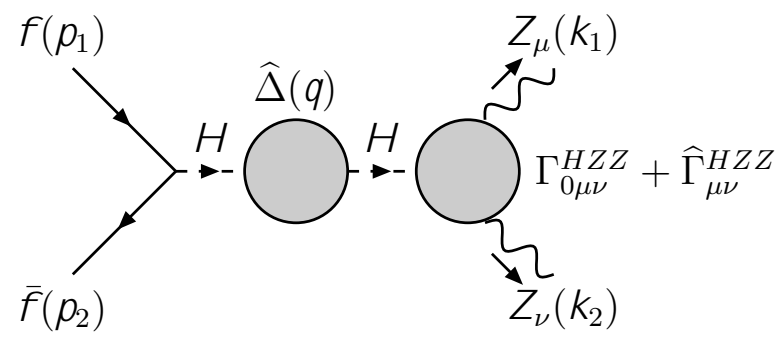

(a)

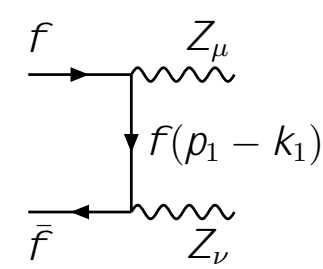

(b)

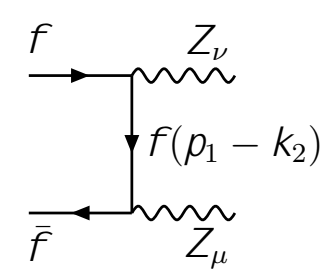

(c)

Figure 7: The Born-improved amplitude for the process $f \bar{f} \rightarrow Z Z$.

contribution of the $Z$ boson-loop reads: $[\underline{\underline{p}}]$

$$
\begin{aligned}
\Pi_{(Z Z)}^{\widehat{H} \widehat{H}}\left(s, \xi_{Q}\right)= & \frac{\alpha_{w}}{32 \pi} \frac{s^{2}}{M_{W}^{2}}\left\{\left(1-4 \frac{M_{Z}^{2}}{s}+\right.\right. \\
& \left.12 \frac{M_{Z}^{4}}{s^{2}}\right) B_{0}\left(s, M_{Z}^{2}, M_{Z}^{2}\right)- \\
& {\left[1+4 \xi_{Q} \frac{M_{Z}^{2}}{s}\right.} \\
& \left.-\left(M_{H}^{2}+4 \xi_{Q} M_{Z}^{2}\right) \frac{M_{H}^{2}}{s^{2}}\right] \\
& \left.\times B_{0}\left(s, \xi_{Q} M_{Z}^{2}, \xi_{Q} M_{Z}^{2}\right)\right\}
\end{aligned}
$$

where $\alpha_{w}=g_{w}^{2} /(4 \pi)$ is the weak fine structure constant and $B_{0}$ is the usual Passarino-Veltman function [15]. The presence of the GFP $\xi_{Q}$ results in bad high energy behaviour and the appearance of unphysical thresholds, as can be verified directly using $\Im m B_{0}\left(s, M^{2}, M^{2}\right)=\theta(s-$ $\left.4 M^{2}\right) \pi\left(1-4 M^{2} / s\right)^{1 / 2}$. Even though to any order in perturbation theory physical amplitudes are GFP-independent, and display only physical thresholds, resumming $\Pi_{(Z Z)}^{\widehat{H}} \widehat{H}\left(s, \xi_{Q}\right)$ will introduce artifacts to the resonant amplitude. Even in the unitary gauge $\left(\xi_{Q} \rightarrow \infty\right)$, where only physical thresholds survive, the $s^{2}$-growth in Eq. (15.1) grossly contradicts the equivalence theorem.

As explained above, in the PT framework a modified one-loop self-energy for the Higgs boson can be constructed, by appending to the conventional self-energy additional propagator-like contributions concealed inside vertices and boxes. These contributions can be identified systematically, by resorting exclusively to elementary Ward identities of the form $k\left(v+a \gamma_{5}\right)=(k+\not p-$ $m)\left(v+a \gamma_{5}\right)-\left(v-a \gamma_{5}\right)(\not p-m)+2 a m \gamma_{5}$, triggered by the longitudinal virtual momenta $k_{\mu}$. Following this procedure, we find the PT Higgs-boson

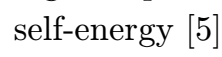

$$
\begin{aligned}
\widehat{\Pi}_{(Z Z)}^{H H}(s)= & \frac{\alpha_{w}}{32 \pi} \frac{M_{H}^{4}}{M_{W}^{2}}\left[1+4 \frac{M_{Z}^{2}}{M_{H}^{2}}-\right. \\
& \left.4 \frac{M_{Z}^{2}}{M_{H}^{4}}\left(2 s-3 M_{Z}^{2}\right)\right] B_{0}\left(s, M_{Z}^{2}, M_{Z}^{2}\right),
\end{aligned}
$$

which is GFP-independent in any gauge fixing scheme, universal $[\overline{9}]$, grows linearly with $s$, and displays physical thresholds only.

To verify that $\widehat{\Pi}_{(Z Z)}^{H H}(s)$ satisfies the optical theorem individually consider the tree-level transition amplitude $\mathcal{T}(Z Z)$ for the process $f\left(p_{1}\right) \bar{f}\left(p_{2}\right) \rightarrow$ $Z\left(k_{1}\right) Z\left(k_{2}\right)$; it is the sum of an $s$ - and a $t$ - channel contribution, denoted by $\mathcal{T}_{s}^{H}(Z Z)$ and $\mathcal{T}_{t}(Z Z)$, respectively, given by

$\mathcal{T}_{s \mu \nu}^{H}(Z Z)=\Gamma_{0 \mu \nu}^{H Z Z} \Delta_{H}(s) \bar{v}\left(p_{2}\right) \Gamma_{0}^{H f \bar{f}} u\left(p_{1}\right)$, 


$$
\begin{aligned}
\mathcal{T}_{t \mu \nu}(Z Z)= & \bar{v}\left(p_{2}\right)\left(\Gamma_{0 \nu}^{Z f \bar{f}} \frac{1}{\not p_{1}+\not k_{1}-m_{f}} \Gamma_{0 \mu}^{Z f \bar{f}}+\right. \\
& \left.\Gamma_{0 \mu}^{Z f \bar{f}} \frac{1}{\not p_{1}+\not k_{2}-m_{f}} \Gamma_{0 \nu}^{Z f \bar{f}}\right) u\left(p_{1}\right) .
\end{aligned}
$$

Here, $s=\left(p_{1}+p_{2}\right)^{2}=\left(k_{1}+k_{2}\right)^{2}$ is the c.m. energy squared, $\Gamma_{0 \mu \nu}^{H Z Z}=i g_{w} M_{Z}^{2} / M_{W} g_{\mu \nu}, \Gamma_{0}^{H f \bar{f}}=$ $-i g_{w} m_{f} /\left(2 M_{W}\right)$ and $\Gamma_{0 \mu}^{Z f \bar{f}}=g_{w} /\left(2 i c_{w}\right) \gamma_{\mu}\left[T_{z}^{f}(1-\right.$ $\left.\left.\gamma_{5}\right)-2 Q_{f} s_{w}^{2}\right]$, with $c_{w}=\sqrt{1-s_{w}^{2}}=M_{W} / M_{Z}$, are the tree-level $H Z Z, H f \bar{f}$ and $Z f \bar{f}$ couplings, respectively, and $Q_{f}$ is the electric charge of the fermion $f$, and $T_{z}^{f}$ its $z$-component of the weak isospin. We then calculate the expression $\left[\mathcal{T}_{s \mu \nu}^{H}(Z Z)\right.$ $\left.+\mathcal{T}_{t \mu \nu}(Z Z)\right] Q^{\mu \rho}\left(k_{1}\right) Q^{\nu \sigma}\left(k_{2}\right)\left[\mathcal{T}_{s \rho \sigma}^{H}(Z Z)+\mathcal{T}_{t \rho \sigma}(Z Z)\right]^{*}$, where $Q^{\mu \nu}(k)=-g^{\mu \nu}+k^{\mu} k^{\nu} / M_{Z}^{2}$ denotes the usual polarization tensor, and isolate its Higgsboson mediated part. To accomplish this, one must first use the longitudinal momenta coming from $Q^{\mu \rho}\left(k_{1}\right)$ and $Q^{\nu \sigma}\left(k_{2}\right)$ in order to extract the Higgs-boson part of $\mathcal{T}_{t}^{\mu \nu}(Z Z)$, i.e.,

$$
\begin{aligned}
\frac{k_{1}^{\mu} k_{2}^{\nu}}{M_{Z}^{2}} \mathcal{T}_{t \mu \nu}(Z Z) & =\mathcal{T}_{P}^{H}+\ldots \\
\mathcal{T}_{P}^{H} & =-\frac{i g_{w}}{2 M_{W}} \bar{v}\left(p_{2}\right) \Gamma_{0}^{H f \bar{f}} u\left(p_{1}\right),
\end{aligned}
$$

where the ellipses denote genuine $t$-channel (not Higgs-boson related) contributions. Then, one must append the piece $\mathcal{T}_{P}^{H} \mathcal{T}_{P}^{H *}$ to the "naive" Higgs-dependent part $\mathcal{T}_{s \mu \nu}^{H}(Z Z) Q^{\mu \rho}\left(k_{1}\right) Q^{\nu \sigma}\left(k_{2}\right)$ $\mathcal{T}_{s \rho \sigma}^{H *}(Z Z)$. Integrating the expression so obtained over the two-body phase space, we finally arrive at the imaginary part of Eq. (5.2i), which is the announced result.

The gauge-invariance of the $S$ matrix imposes tree-level Ward identities on the unrenor-

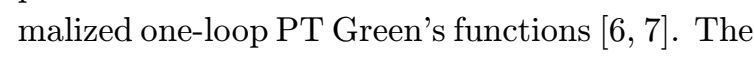
requirement that the same Ward identities should be maintained after renormalization gives rise to important QED-type relations for the renormalization constants of the theory. Specifically, we find

$$
\begin{aligned}
& \widehat{Z}_{W}=\widehat{Z}_{g_{w}}^{-2}, \quad \widehat{Z}_{Z}=\widehat{Z}_{W} \widehat{Z}_{c_{w}}^{2}, \\
& \widehat{Z}_{H}=\widehat{Z}_{W}\left(1+\delta M_{W}^{2} / M_{W}^{2}\right),
\end{aligned}
$$

where $\widehat{Z}_{W}, \widehat{Z}_{Z}$, and $\widehat{Z}_{H}$ are the wave-function renormalizations of the $W, Z$ and $H$ fields, respectively, $\widehat{Z}_{g_{w}}$ is the coupling renormalization, and $\widehat{Z}_{c_{w}}=\left(1+\delta M_{W}^{2} / M_{W}^{2}\right)^{1 / 2}\left(1+\delta M_{Z}^{2} / M_{Z}^{2}\right)^{-1 / 2}$. The renormalization of the bare resummed Higgsboson propagator $\hat{\Delta}^{H, 0}(s)$ proceeds as follows:

$$
\begin{aligned}
\hat{\Delta}^{H, 0}(s) & =\left[s-\left(M_{H}^{0}\right)^{2}+\widehat{\Pi}^{H H, 0}(s)\right]^{-1} \\
& =\widehat{Z}_{H}\left[s-M_{H}^{2}+\widehat{\Pi}^{H H}(s)\right]^{-1} \\
& =\widehat{Z}_{H} \hat{\Delta}^{H}(s)
\end{aligned}
$$

with $\left(M_{H}^{0}\right)^{2}=M_{H}^{2}+\delta M_{H}^{2}$. The renormalized Higgs-boson mass $M_{H}^{2}$ may be defined as the real part of the complex pole position of $\hat{\Delta}^{H}(s)$. Employing the relations in Eq. (5.5. the universal quantity

$$
\begin{aligned}
\widehat{R}^{H, 0}(s) & =\frac{\left(g_{w}^{0}\right)^{2}}{\left(M_{W}^{0}\right)^{2}} \hat{\Delta}^{H, 0}(s)=\frac{g_{w}^{2}}{M_{W}^{2}} \hat{\Delta}^{H}(s) \\
& =\widehat{R}^{H}(s)
\end{aligned}
$$

is invariant under the renormalization group. This important universal property of the Higgs boson is true for non-Abelian gauge theories with spontaneous symmetry breaking (SSB), but does not hold in general.

An additional, highly non-trivial constraint, must be imposed on resummed amplitudes; they have to obey the (generalized) equivalence theorem (GET), which is known to be satisfied before resummation, order by order in perturbation theory. For the specific example of the amplitude $\mathcal{T}(Z Z)=\mathcal{T}_{s}^{H}+\mathcal{T}_{t}$, the GET states that

$$
\begin{aligned}
\mathcal{T}\left(Z_{L} Z_{L}\right)= & -\mathcal{T}\left(G^{0} G^{0}\right)-i \mathcal{T}\left(G^{0} z\right) \\
& -i \mathcal{T}\left(z G^{0}\right)+\mathcal{T}(z z),
\end{aligned}
$$

where $Z_{L}$ is the longitudinal component of the $Z$ boson, $G^{0}$ is its associated would-be Goldstone boson, and $z^{\mu}(k)=\varepsilon_{L}^{\mu}(k)-k^{\mu} / M_{W}$ is the energetically suppressed part of the longitudinal polarization vector $\varepsilon_{L}^{\mu}$. It is crucial to observe, however, that already at the tree level, the conventional $s$ - and $t$ - channel sub-amplitudes $\mathcal{T}_{s}^{H}$ and $\mathcal{T}_{t}$ fail to satisfy the GET individually. To verify that, one has to calculate $\mathcal{T}_{s}^{H}\left(Z_{L} Z_{L}\right)$, using explicit expressions for the longitudinal polarization vectors, and check if the answer obtained is equal to the Higgs-boson mediated $s$-channel part of the LHS of Eq. (5.8). In particular, in the c.m. system, we have $z^{\mu}\left(k_{1}\right)=\varepsilon_{L}^{\mu}\left(k_{1}\right)-$ $k_{1}^{\mu} / M_{Z}=-2 M_{Z} k_{2}^{\mu} / s+\mathcal{O}\left(M_{Z}^{4} / s^{2}\right)$, and exactly analogous expressions for $z^{\mu}\left(k_{2}\right)$. The residual 
vector $z^{\mu}(k)$ has the properties $z_{\mu} k^{\mu}=-M_{Z}$ and $z^{2}=0$. After a straightforward calculation, we obtain $\mathcal{T}_{s}^{H}\left(Z_{L} Z_{L}\right)=-\mathcal{T}_{s}^{H}\left(G^{0} G^{0}\right)-i \mathcal{T}_{s}^{H}\left(z G^{0}\right)-$ $i \mathcal{T}_{s}^{H}\left(G^{0} z\right)+\mathcal{T}_{s}^{H}(z z)-\mathcal{T}_{P}^{H}$, where

$$
\mathcal{T}_{s}^{H}\left(G^{0} G^{0}\right)=\Gamma_{0}^{H G^{0} G^{0}} \Delta_{H}(s) \bar{v}\left(p_{2}\right) \Gamma_{0}^{H f \bar{f}} u\left(p_{1}\right),
$$

$$
\begin{aligned}
\mathcal{T}_{s}^{H}\left(z G^{0}\right)+\mathcal{T}_{s}^{H}\left(G^{0} z\right)= & {\left[z^{\mu}\left(k_{1}\right) \Gamma_{0 \mu}^{H Z G^{0}}+\right.} \\
& \left.z^{\nu}\left(k_{2}\right) \Gamma_{0 \nu}^{H G^{0} Z}\right] \times \\
& \Delta_{H}(s) \bar{v}\left(p_{2}\right) \Gamma_{0}^{H f \bar{f}} u\left(p_{1}\right) \\
\mathcal{T}_{s}^{H}(z z)= & z^{\mu}\left(k_{1}\right) z^{\nu}\left(k_{2}\right) \mathcal{T}_{s \mu \nu}^{H}(Z Z)
\end{aligned}
$$

with $\Gamma_{0}^{H G^{0} G^{0}}=-i g_{w} M_{H}^{2} /\left(2 M_{W}\right)$ and $\Gamma_{0 \mu}^{H Z G^{0}}=$ $-g_{w}\left(k_{1}+2 k_{2}\right)_{\mu} /\left(2 c_{w}\right)$. Evidently, the presence of the term $\mathcal{T}_{P}^{H}$ prevents $\mathcal{T}_{s}^{H}\left(Z_{L} Z_{L}\right)$ from satisfying the GET. This is not surprising however, since an important Higgs-boson mediated $s$-channel part has been omitted. Specifically, the momenta $k_{1}^{\mu}$ and $k_{2}^{\nu}$ stemming from the leading parts of the longitudinal polarization vectors $\varepsilon_{L}^{\mu}\left(k_{1}\right)$ and $\varepsilon_{L}^{\nu}\left(k_{2}\right)$ extract such a term from $\mathcal{T}_{t}\left(Z_{L} Z_{L}\right)$. Just as happens in Eq. $(\overline{5} . \overline{4})$, this term is precisely $\mathcal{T}_{P}^{H}$, and must be added to $\mathcal{T}_{s}^{H}\left(Z_{L} Z_{L}\right)$, in order to form a well-behaved amplitude at very high energies. In other words, the amplitude $\widehat{\mathcal{T}}_{s}^{H}\left(Z_{L} Z_{L}\right)=$ $\mathcal{T}_{s}^{H}\left(Z_{L} Z_{L}\right)+\mathcal{T}_{P}^{H}$ satisfies the GET independently (cf. Eq. (5.8) ). In fact, this crucial property persists after resummation. Indeed, as shown in Fig.7 the resummed amplitude $\overline{\mathcal{T}}_{s}^{H}\left(Z_{L} Z_{L}\right)$ may be constructed from $\mathcal{T}_{s}^{H}\left(Z_{L} Z_{L}\right)$ in Eq. (5.3i), if $\Delta_{H}(s)$ is replaced by the resummed Higgs-boson propagator $\hat{\Delta}^{H}(s)$, and $\Gamma_{0 \mu \nu}^{H Z Z}$ by the expression $\Gamma_{0 \mu \nu}^{H Z Z}+\widehat{\Gamma}_{\mu \nu}^{H Z Z}$, where $\widehat{\Gamma}_{\mu \nu}^{H Z Z}$ is the one-loop $H Z Z$ vertex calculated within the $\mathrm{PT}[[\bar{n} \mid]$. It is then straightforward to show that the Higgs-mediated amplitude $\widetilde{\mathcal{T}}_{s}^{H}\left(Z_{L} Z_{L}\right)=\overline{\mathcal{T}}_{s}^{H}\left(Z_{L} Z_{L}\right)+\mathcal{T}_{P}^{H}$ respects the GET individually; to that end we only need to employ the following tree-level-type PT WI's:

$$
\begin{aligned}
& k_{2}^{\nu} \widehat{\Gamma}_{\mu \nu}^{H Z Z}\left(q, k_{1}, k_{2}\right)+i M_{Z} \widehat{\Gamma}_{\mu}^{H Z G^{0}}\left(q, k_{1}, k_{2}\right)= \\
& -\frac{g_{w}}{2 c_{w}} \widehat{\Pi}_{\mu}^{Z G^{0}}\left(k_{1}\right), \\
& k_{1}^{\mu} \widehat{\Gamma}_{\mu}^{H Z G^{0}}\left(q, k_{1}, k_{2}\right)+i M_{Z} \widehat{\Gamma}^{H G^{0} G^{0}}\left(q, k_{1}, k_{2}\right)= \\
& -\frac{g_{w}}{2 c_{w}}\left[\widehat{\Pi}^{H H}\left(q^{2}\right)+\widehat{\Pi}^{G^{0} G^{0}}\left(k_{2}^{2}\right)\right], \\
& k_{1}^{\mu} k_{2}^{\nu} \widehat{\Gamma}_{\mu \nu}^{H Z Z}\left(q, k_{1}, k_{2}\right)+M_{Z}^{2} \widehat{\Gamma}^{H G^{0} G^{0}}\left(q, k_{1}, k_{2}\right)=
\end{aligned}
$$

$$
\frac{i g_{w} M_{Z}}{2 c_{w}}\left[\widehat{\Pi}^{H H}\left(q^{2}\right)+\widehat{\Pi}^{G^{0} G^{0}}\left(k_{1}^{2}\right)+\widehat{\Pi}^{G^{0} G^{0}}\left(k_{2}^{2}\right)\right]
$$

where $\widehat{\Gamma}_{\mu}^{H Z G^{0}}$ and $\widehat{\Gamma}^{H G^{0} G^{0}}$ are the one-loop PT $H Z G^{0}$ and $H G^{0} G^{0}$ vertices, respectively. In this derivation, one should also make use of the PT WI involving the $Z G^{0}$ - and $G^{0} G^{0}$ - self-energies: $\widehat{\Pi}_{\mu}^{Z G^{0}}(k)=-i M_{Z} k_{\mu} \widehat{\Pi}^{G^{0} G^{0}}\left(k^{2}\right) / k^{2}$.

The partial running widths for the Higgs boson have been first calculated at one-loop in [05]; they are given by:

$$
\begin{aligned}
\Im m \widehat{\Pi}_{(W W)}(s)= & \frac{\alpha_{w}}{16} \frac{M_{H}^{4}}{M_{W}^{2}}\left[1+4 \frac{M_{W}^{2}}{M_{H}^{2}}-\right. \\
& \left.4 \frac{M_{W}^{2}}{M_{H}^{4}}\left(2 s-3 M_{W}^{2}\right)\right] \beta_{W} \theta\left(s-4 M_{W}^{2}\right), \\
\Im m \widehat{\Pi}_{(Z Z)}(s)= & \frac{\alpha_{w}}{32} \frac{M_{H}^{4}}{M_{W}^{2}}\left[1+4 \frac{M_{Z}^{2}}{M_{H}^{2}}-\right. \\
& \left.4 \frac{M_{Z}^{2}}{M_{H}^{4}}\left(2 s-3 M_{Z}^{2}\right)\right] \beta_{Z} \theta\left(s-4 M_{Z}^{2}\right),
\end{aligned}
$$

$\Im m \widehat{\Pi}_{(F F)}(s)=N_{F} \frac{\alpha_{w}}{8} \frac{m_{F}^{2}}{M_{W}^{2}} s \beta_{F}^{3} \theta\left(s-4 m_{F}^{2}\right)$,

$\Im m \widehat{\Pi}_{(H H)}(s)=\frac{9 \alpha_{w}}{32} \frac{M_{H}^{4}}{M_{W}^{2}} \beta_{H} \theta\left(s-4 M_{H}^{2}\right)$.

In the above formula we denote by $F$ the various fermionic flavours appearing inside the quantum loops, i.e. $F \in\{e, \mu, \tau, u, d, c, s, t, b\} . N_{F}=1$ for leptons, and $N_{F}=3$ for quarks. In the case of a heavy Higgs boson the channels which dominate numerically are the $W W, Z Z$ and $t$.

\section{Conclusions}

We have seen that, at one-loop order in perturbation theory the Breit-Wigner resummation formalism can be extended to the case of nonAbelian gauge theories, provided that one resorts to the pinch technique rearrangement of the physical amplitude. To accomplish this one needs invoke only the full exploitation of the elementary Ward-identities of the theory, in conjunction with unitarity, analyticity, and renormalization group invariance.

From the phenomenological point of view the above framework enables the construction of Born- 
improved amplitudes in which all relevant physical information has been encoded. This in turn will be very useful for the detailed study of the physical properties of particles, most importantly the correct extraction of their masses, widths, and line shapes.

It would be very interesting to extend the formalism described in this paper to higher orders. In addition, one would like to reach a formal understanding of the underlying mechanism, which at present can only be diagrammatically exposed. We hope to be able to return to these issues in the near future.

\section{Acknowledgements}

The research presented here has been carried out in collaboration with Apostolos Pilaftsis, whom I thank for countless stimulating discussions spanning a period of several years. The contributions of Eduardo de Rafael and Jay Watson have been instrumental for my present understanding of the subject. I also like to thank Fawzi Boudjema, Costas Kounnas, Kostas Philippides, Rob Pisarski, and Raymond Stora, for various useful discussions. This work has been funded by a Marie Curie Fellowship (TMR-ERBFMBICT 972024).

\section{References}

[1] A. Sirlin, Phys. Rev. Lett. 67, 2127 (1991); Phys. Lett. B267, 240 (1991); R. G. Stuart, Phys. Lett. B262, 113 (1991); Phys. Lett. B272, 353 (1991); Phys. Rev. Lett. 70, 3193 (1993); M. Nowakowski and A. Pilaftsis, Z. Phys. C60, 121 (1993); A. Aeppli, G.J. van Oldenborgh, and D. Wyler, Nucl. Phys. B428, 126 (1994); H. Veltman, Z. Phys. C62, 35 (1994); U. Baur and D. Zeppenfeld, Phys. Rev. Lett. 75, 1002 (1995); M. Beuthe, R. Gonzalez Felipe, G. Lopez Castro, and J. Pestieau, Nucl. Phys. B498, 55 (1997); L. Maiani and M. Testa, Annals Phys. 263, 353 (1998).

[2] D.A. Dicus and V.S. Mathur, Phys. Rev. D7, 3111 (1973); W.W. Repko and C.J. Suchyta, Phys. Rev. Lett. 62, 859 (1989); S. Dawson and S. Willenbrock, Phys. Rev. D40, 2880 (1989); A. Dobado, Phys. Lett. B237, 457 (1990); G. Valencia and S. Willenbrock, Phys. Rev.
D46, 2247 (1992); M.H. Seymour, Phys. Lett. B354, 409 (1995); K. Philippides and A. Sirlin; Phys. Lett. B367, 377 (1996); A. Ghinculov and J.J. van der Bij, Nucl. Phys. B482, 59 (1996); K. Philippides and A. Sirlin; Nucl. Phys. B477 59, (1996); D. Wackeroth and W. Hollik, Phys. Rev. D55, 6788 (1997); M. Kuroda and D. Schildknecht, Nucl. Phys. B531, 24 (1998); M. Passera and A. Sirlin, Phys. Rev. D58 (1998) 113010; B. A. Kniehl and A. Sirlin Phys. Rev. Lett. 81, 1373 (1998).

[3] J.M. Cornwall, D.N. Levin, and G. Tiktopoulos, Phys. Rev. D10, 1145 (1974); E11, 972 (1975); C.E. Vayonakis, Lett. Nuovo Cim. 17, 383 (1976); M.S. Chanowitz and M.K. Gaillard, Nucl. Phys. B261, 379 (1985); G.J. Gounaris, R. Kögerler, and H. Neufeld, Phys. Rev. D34, 3257 (1986).

[4] J. Papavassiliou and A. Pilaftsis, Phys. Rev. Lett. 75, 3060 (1995); Phys. Rev. D53, 2128 (1996); Phys. Rev. D54, 5315 (1996);

[5] J. Papavassiliou and A. Pilaftsis, Phys. Rev. Lett. 80, 2785 (1998); Phys. Rev. 58, (1998) 53002 .

[6] J.M. Cornwall, in Proceedings of the FrenchAmerican Seminar on Theoretical Aspects of Quantum Chromodynamics, Marseille, France, 1981, edited J.W. Dash (Centre de Physique Théorique, Marseille, 1982); Phys. Rev. D26, 1453 (1982).

[7] J.M. Cornwall and J. Papavassiliou, Phys. Rev. D40, 3474 (1989); J. Papavassiliou, Phys. Rev. D 41, 3179 (1990); G. Degrassi and A. Sirlin, Phys. Rev. D 46, 3104 (1992).

[8] J. Papavassiliou, E. de Rafael, and N.J. Watson, Nucl. Phys. B503, 79 (1997).

[9] N.J. Watson, Phys. Lett. B349, 155 (1995).

[10] N.J. Watson, Nucl. Phys. B494, 388 (1997).

[11] C. Becchi, A. Rouet, and R. Stora, Ann. Phys. (NY) 98, 287 (1976).

[12] J. Papavassiliou, Phys. Rev. D50, 5958 (1994).

[13] See for example L.F. Abbott, Nucl. Phys. B185, 189 (1981), and references therein.

[14] A. Denner, S. Dittmaier, and G. Weiglein, Phys. Lett. B333, 420 (1994), Nucl. Phys. B440, 95 (1995); S. Hashimoto, J. Kodaira, Y. Yasui, and K. Sasaki, Phys. Rev. D50, 7066 (1994);

[15] G. 't Hooft and M. Veltman, Nucl. Phys. B153, 365 (1979); G. Passarino and M. Veltman, Nucl. Phys. B160, 151 (1979). 